\title{
Production Planning with Hot Section Life Prediction for Optimum Gas Turbine Management
}

\author{
Giovanni Cerri $^{1} \quad$ Marco Gazzino $^{1} \quad$ Fabio Botta $^{1} \quad$ Coriolano Salvini $^{1}$ \\ ${ }^{1}$ Department of Mechanical and Industrial Engineering \\ ROMA TRE University \\ Via della Vasca Navale, 79 - 00146 Roma (ITALY) \\ Tel. +39-06-55173251/ Fax +39-06-5593732 \\ cerri@uniroma3.it
}

\begin{abstract}
The power production planning problem has been deeply investigated. Maintenance management and load allocation problems have been assumed as crucial aspects for achieving maximum plant profitability. Modeling of life consumption of hot section components has been considered as one of the key feature necessary to simulate the plant behaviour.

The approach takes market scenarios, as well as actual status and performance of plant components into account. A supervisor algorithm provides the operating parameters needed to establish each plant loading. Economic implications related to maintenance strategies including postponement or anticipation of maintenance interventions are investigated and results obtained by the numerical simulation are presented and widely discussed.
\end{abstract}

\section{NOMENCLATURE}

D vector of plant model constraints and inequalities

$\Delta \mathrm{t} \quad$ time interval

$f \quad$ life consumption ratio

$\mathrm{F} \quad$ vector of plant model equations

G profit

$\mathrm{L}_{\mathrm{A}} \quad$ actual life

$\mathrm{L}_{\mathrm{n}} \quad$ nominal life

$\mathrm{L}_{\mathrm{U}} \quad$ useful life

$\mathrm{P} \quad$ power output

$\mathrm{p}_{\mathrm{EL}} \quad$ power sale price

$\mathrm{T} \quad$ planning period

$\mathrm{T}_{\mathrm{b}} \quad$ blade temperature

$\mathrm{T}_{\mathrm{c}} \quad$ coolant temperature

$\mathrm{T}_{\mathrm{g}} \quad$ gas temperature

$\mathrm{u} \quad$ vector of component's status factors

$\mathrm{V} \quad$ creep model input vector

$\mathrm{Z} \quad$ vector of plant process and state variables

\section{Greek letters}

\section{$\varepsilon \quad$ global cooling efficiency}

$\xi$ degrees of freedom; vector of quantities specifying the plant operating point

\section{Subscripts and Superscripts}

\section{CT}

EQ

GB

TH

\section{centrifugal}

equivalent

gas bending

thermal

Presented at International Gas Turbine Congress 2007 Tokyo, Dec. 2-7, Tokyo, Japan

Manuscript received on Oct. 21, 2007

Review completed on Jul. 31, 2008

\section{INTRODUCTION}

Considerable technological efforts have been recently devoted to increasing efficiencies of energy conversion systems, in an attempt to achieve better use of resources within environmental compliance.

Gas Turbines (GTs) have had a key role in this process, by dramatically increasing their power and efficiency levels over the last 25 years. The application of aero-derivative engines has provided GT-based power plants with high compression ratio units and it has led combustor outlet temperatures over $1300^{\circ} \mathrm{C}$ to be ordinary. Thermal efficiencies of simple-cycle solutions are usually bounded in the $35 \div 40 \%$ (LHV) range, with specific power around $400 \mathrm{~kW} \cdot \mathrm{kg}_{\mathrm{AIR}}{ }^{-1} \cdot \mathrm{s}(50 \mathrm{~Hz})$.

Such developments have also had a positive impact on combined gas-steam plants, making them the solution considered as the more convenient for electricity generation. Reasons for such popularity are excellent operational flexibility and maintainability, relatively low investment costs and high electrical efficiencies, which altogether lead to competitive electricity production costs and reduced emissions, including $\mathrm{CO}_{2}$.

Presently, 60\% (LHV) and above efficiencies are practicable with combined cycle power plants. Moreover, specific power easily overcomes the frontier of $600 \mathrm{~kW} \cdot \mathrm{kg}_{\mathrm{AIR}}{ }^{-1} \cdot \mathrm{s}(50 \mathrm{~Hz})$. Due to the limitations of the steam turbine when starting up from cold and to the large thermal inertia of Heat Recovery Steam Generator (HRSG), combined cycle power plants show less operating flexibility compared to the simple cycle ones.

The increasing concern for $\mathrm{CO}_{2}$ emissions pushes for improvements in power plant efficiencies, which are important are important both for short term and for long term solutions of reducing $\mathrm{CO}_{2}$ (Beér, 2006). In the short term, efficiency increase is the only way to achieve an immediate reduction in $\mathrm{CO}_{2}$ emissions. In the case of long-term solutions, such as oxygen-blown pressurised gasification of coal, oxy-fuel combustion or chemical absorption, the high efficiency allows mitigating energy penalties associated to Carbon Capture and Sequestration (CCS) technologies.

With the purpose to realize a highly efficient energy conversion, several cycle and layouts have been proposed. Future developments include the integration of gas turbines into high-level technology systems, such as combined-cycles with pressurized fluidized bed combustors, humid cycles or hybrid power systems, which utilize fuel cells and gas turbines together.

According to the above considerations, it is clear that GTs are playing and will still be playing for many years a relevant role in the field of electricity generation. As a result, several studies have been published concerning maintenance management and investigating possibilities for a better exploitation of gas turbines during their useful life. Cerri et al. (2005-a) proposed an inverse method- 
ology for actual status recognition of GT based power plants from data collected by the plant monitoring system. Benvenuti (2003), Sampath and Singh (2003) suggested the application of innovative and advanced fault diagnostic techniques, in order to achieve improved operational availabilities and service performances. Silva et al. (2003) showed the benefits related to an optimized scheduling of cleaning time-intervals on GT compressor. Lowden at al. (2006) proposed a methodology to predict blade remaining useful life and assess on-line statuses of components for condition-based maintenance purposes.

It is generally recognized that operating a GT over its recommended useful life can be done rescheduling the maintenance according to the effective life of Hot Gas Path Parts (HGPPs). Thus, life assessment tools for hot section components are required. Several life prediction approaches have been proposed (Cunha et al., 2005; Beachkofski and Grandhi, 2004; Wallace and Mavris, 2003; Liu et al., 2001). Since maintenance costs usually include refurbishment and substitution of components, a low accuracy in assessing the actual useful life causes over-costs, because components tend to be replaced before having finished their serviceable life. Such considerations become dramatically relevant in the management of a GT, which has hot-section components exposed to severe operating conditions, causing damage rate to depend on the power level exponentially. Hence, the deterioration of high temperature components is one of the most important factors to be considered for an effective maintenance strategy and an optimized production planning.

This paper mainly focuses on the planning algorithm, including plant simulator and deterioration models. It also shows how the application of a reliable damage model in a production-planning problem could lead to improved machine exploitation through an effective maintenance scheduling.

\section{POWER PLANT DESCRIPTION}

A pool of five combined-cycle power plants is adopted for the simulation. Each plant is based on a twin shaft aero-derivative GT (LM6000-PA), driving an electric generator and discharging exhaust gases through a two-pressure level steam generator, which provides steam to a condensation steam turbine with $10 \mathrm{MW}$ as installed capacity. All units are fed by natural gas and all steam produced by the Heat Recovery Steam Generator (HRSG) expands in the steam turbine and no steam is bled for thermal power production. The steam section of the plant is equipped with an air cooled condenser.

Equipments and layouts of each plant of the pool are all the same. Due to different geographical regions where plants are installed and the different local weather conditions, as well as to the different operational history of plant components, a diversification in boundary conditions and initial statuses of components can occur.

\section{SIMULATION METHODOLOLGY}

In order to perform the production planning of a pool of power plants, a planning algorithm has been developed, based on Genetic Algorithm techniques. Since the planning algorithm needs machine parameters and thermodynamic variables to be evaluated at each time instant, a numerical simulator of the plant has been produced. If an accurate production planning is required, plant performance deterioration, as well as accumulated damage of components must be taken into account.

According to the needs of the planning procedure, the plant simulator includes dedicated modules taking deterioration of components into account and assessing residual useful life of HGPPs.

Models applied for simulation of plant components, as well as the methodology adopted to account for performance variations related to deterioration of components are briefly discussed in the following. Then, the proposed creep model for hot section life-assessment is described and discussed.

\section{Plant model}

The overall plant model is based on a modular description of components. Each module could represent a single component or a group of them. Modules are composed by sets of linear and non-linear equations, including the conservation of Mass, Energy, Momentum, Entropy, as well as constitutive and auxiliary equations, which describe other phenomena such as heat transfer, pressure loss or equipment specifications and control rules.

Accordingly, steady state behavior of the plants and the pool is described by a set of equations $\mathbf{F}$ :

$$
F(\mathbf{u}, \mathbf{z}, \xi)=\mathbf{0}
$$

and inequalities $\boldsymbol{D}$ :

$$
D(\mathbf{u}, \mathbf{z}, \xi)=\mathbf{0}
$$

$\mathbf{u}$ giving the state of the system (fouling, performance deterioration, etc.), $\mathbf{z}$ being the vector of the unknown variables, $\xi$ being the vector of Degrees Of Freedom (DOFs). The set of conditions D constrains the domain of existence for the solution.

Each module simulates the off-design behaviour of the component and carries out the relationship between input and output variables. A simultaneous solution method has been adopted, performing the minimization of a plant objective function defined as a weighted sum of the partial objective functions given as outputs of modules. A simultaneous solution method developed by Cerri (1996) has been applied to get the solution.

Component models include databases (DBs) containing geometries, shapes and architectures, with the corresponding related correlations (e.g. profile cascade features and related losses and deviations, finned tube bundles features and related heat transfer coefficients). Such DBs are adopted to select arrangements on the basis of manufacturer information or default choices.

\section{Deterioration of plant components}

Performance of plant components changes during their life due to phenomena like fouling, corrosion, erosion of parts, etc., affecting actual behaviour of plant units. This means that going on with operations different machines and apparatuses exist at each time instant. Since characteristic curves of components and performance maps are continuously changing, they need to be continuously re-established within the model if an accurate plant operation management is to be accomplished.

In order to take such changes into account, models contain polynomial Actuality Functions (AFs) which adapt N\&C real component models to the continuously changing actual component behaviours. AFs are established to modify component performance in terms of work transfer or heat transfer, dissipative phenomena and effective flow functions. The assumption is that component performance degradation implies AFs being less than one.

In the adopted models, the choice of using global AFs has been made. Such functions are necessary to replicate behaviours of plant components and to describe their present performance. Cerri et al. (2006) report more details about definition and application of AFs.

\section{Neural plant simulator}

Since direct application of physical and empirical models to a problem that requires iterative calculations can lead to a quite long calculation time, alternative simulation procedures must be considered. In order to perform low CPU occupancy and to get the solution in short time, ANN techniques have been chosen and applied. The purpose has been to approximate a stated input-output map that represents the behaviour of the plant.

The plant model has been utilized to generate the database needed for ANN training and testing. Then, single-layer feedforward networks have been trained with backpropagation algorithm and a parametric simulator of the plant has been produced. Cerri et al. (2005-b) extensively discussed neural methodologies to speed up calculations related to heat and power cogeneration plants. 


\section{CREEP MODEL}

In order to perform hot section life calculation, a creep model has been developed and applied to first and second stage buckets of HPT. The modelled physical system is composed of a row of blades jointed on a rotating disk and surrounded by a hot gas stream, with a bleeding of high-pressure air taken from the compressor and passing through the blade inside cooling passages.

The most elaborate models traditionally used to evaluate life consumption due to creep are based on time consuming finite elements procedures and result in very computationally expensive tasks. The proposed creep model has been developed specifically to be used in a production planning procedure (where fast calculations are essential). Accordingly, a one-dimensional approach has been adopted.

The presented creep model assumes lumped quantities along the hub-tip centroids line. Actions referred to centrifugal, gas bending and thermal stresses have been considered as main stress sources. By simulating thermal and mechanical loadings on turbine buckets and calculating the corresponding equivalent stress, the creep model assesses residual life using parameterized data. The equivalent uniaxial stress has been evaluated combining thermal and mechanical stresses by means of the well known Von-Mises formula.

The interaction between creep and corrosion is simulated through a physical reduction of section areas, which causes a corresponding increase in primary stress levels that occurs as airfoil thickness decreases. Hot corrosion behaviour has been assumed to be pseudo-linear. The resulting increased stress levels are taken into account by adjusting the equivalent stress according to the reduction in cross section areas.

Gas path flow conditions, such as temperatures, pressures, velocities, flow angles, etc., must be previously known, since they are treated as input for the creep model. Hence, a physical-empirical model developed by Cerri et al. (2006) for the LM6000-PA gas turbine has been applied to perform these calculations. The model adopts a modular approach, simulating the behaviour of main GT components. Component specifications of the LM6000-PA have been properly introduced in the model and the simulator has been calibrated with empirical data resulting from real plant operations.

A complete description of the creep model is reported by Gazzino (2005) and by Cerri et al. (2006). In the following, details on blade temperature and mechanical load calculation are reported.

\section{Blade temperature}

Blade has been divided into seven finite elements, each one identified by a node. Accordingly, differential equations describing heat transfer can be written in terms of one-dimensional finite differences. Since heat transfer related to thermal radiation has been neglected, heat transfer equations associated to each node take only

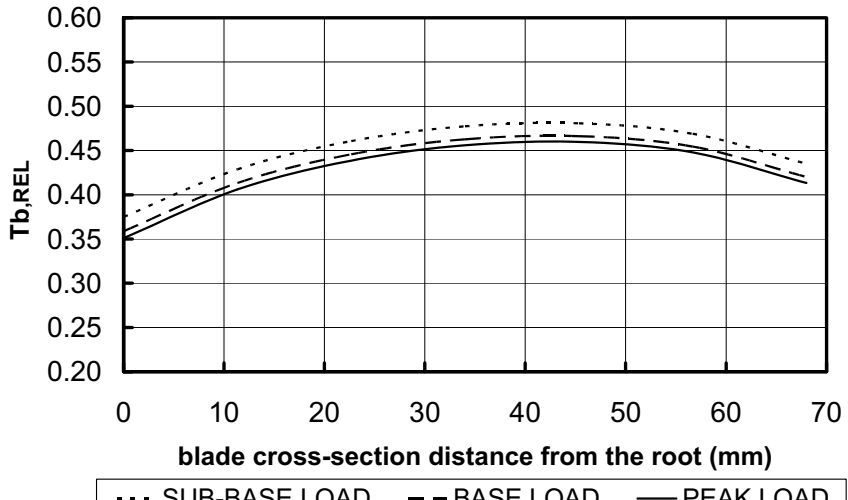

Fig. 1 - Blade relative temperature distributions convection and conduction into account.

A simplified cooling scheme has been adopted, with a single cooling passage, so that cooling air goes from root to tip straightforward. A global cooling efficiency $\varepsilon_{\mathrm{j}}$ has been introduced, including all specific cooling aspects (convection, impingement, film cooling, architecture of the channel, area and spacing of injection holes and so on). Global cooling efficiency is defined as follows:

$$
\varepsilon_{j}=\frac{T_{g, j}-T_{b, j}}{T_{g, j}-T_{c, j+1}}
$$

$T_{g}$ being gas temperature, $T_{b}$ being blade temperature and $T_{c}$ being coolant temperature. All quantities are related to the $\mathrm{j}$-th section. Blade temperature $T_{b, j}$ associated to the $\mathrm{j}$-th node is an effective mean-temperature, representing the actual temperature distribution on each cross section.

Fig. 1 shows relative blade temperature distributions, $T_{b, R E L}$ being defined as follows:

$$
T_{b, R E L}=\frac{T_{b, j}-T_{c, j}}{T_{g, j}-T_{c, j}}
$$

All figures refer to HPT first stage rotor blades of the LM6000 gas turbine manufactured by General Electric. All curves are evaluated at ISO ambient conditions.

\section{Mechanical load calculation}

The creep model takes centrifugal, gas bending and thermal stresses into account and a plane state of stress has been adopted. Centrifugal actions lead to tensile stresses, bending stresses and torsional stresses. Bending and torsional stresses arise when the centroids of the blade cross-sections at different radii do not lie on a radial line and, usually, they are small enough to be neglected. As a result, tensile stress contribution is the most relevant one and the only considered in the simulation.

The value of centrifugal tensile stress at a generic blade cross-section is given by:

$$
\sigma_{c t}(r)=\frac{\rho_{b} \omega^{2}}{A(r)} \cdot \int_{r}^{r_{T}}\left(A\left(r^{\prime}\right) \cdot r^{\prime}\right) d r^{\prime}
$$

$\rho_{b}$ being blade material density, $\omega$ being angular velocity, $A$ being the area of blade cross-section, $r$ being radius, $r_{T}$ being the tip radius and $r$ ' being an integration variable.

In order to simplify centrifugal stress calculation, a linear tapering with radius has been adopted, so as to express centrifugal stress in terms of a cross section ratio At/Ar. A high accuracy in evaluating centrifugal stress can be expected, since all assumptions made are consistent with the reduced height of turbine blades in early stages, as well as with their slight tapering and twisting.

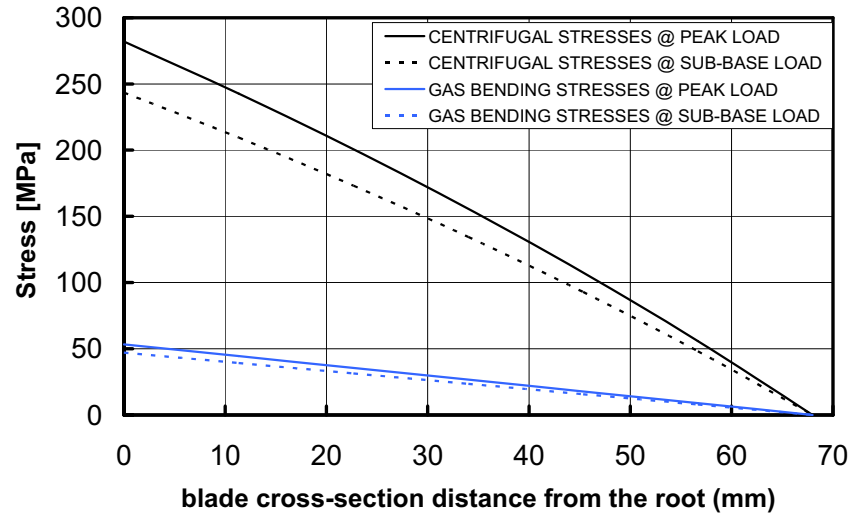

Fig. 2 - Centrifugal and gas bending stress curves 
As mentioned above, gas-bending stresses have also been evaluated. These arise from a gas bending moment, which can be resolved into two components, respectively connected with the change in angular momentum of the flow in the tangential direction and with the pressure drop through the stage. Gas bending moment $M_{a}$ about the axial direction (due to flow deviation) is the greater contributing moment and it is responsible for useful torque, thus only this component has been taken into account. $M_{a}$ is a function of the gas flow rate and gas deflection as follows:

$$
M_{a}=\int_{\text {root }}^{t i p} \rho_{g} c_{a} \cdot\left(c_{t, 1}-c_{t, 2}\right) \cdot s r d r
$$

$\rho_{b}$ being gas density, $s$ being pitch, $r$ being radius, $c_{a}$ being gas axial-velocity, $c_{t, 1}$ and $c_{t, 2}$ being tangential velocities at inlet and outlet sections respectively.

Fig. 2 shows centrifugal and gas bending stress curves evaluated by the model. Such curves are evaluated at ISO ambient conditions and refer to HPT first stage rotor blades of the LM6000 gas turbine (General Electric). As radial temperature distribution on the blade is known, thermal stresses are calculated depending on thermal gradients, linear thermal expansion coefficients and Young's modulus, introduced as functions of metal temperature.

\section{POWER PRODUCTION PLANNING}

The problem of planning the production of a pool of power plants is stated as determining arrangement of the pool at each characteristic instant $t_{k}$ and performing allocation of loads to the units, so as to maximize earnings during the whole planning period $T$. Such optimization must be performed according to initial conditions, as well as to time distributions of ambient and economic quantities, such as ambient temperature, pressure and relative humidity distributions, fuel price distribution, distribution of supply costs and power sale-price distribution.

Production planning is a constrained problem, including both economic (related to the market) and technical (related to the plant) constraints, such as plant availabilities, minimum and maximum power output provided by each unit, minimum up/down time, requested reservoir level and power to be produced according to the energy demand scheduling.

The solution of the production-planning problem can be reached by finding the DOFs distribution $\xi^{*}(t)$ that satisfies the following condition, which is subject to initial and boundary conditions:

$$
\xi *(t) \mid \max \left\{G=\int_{t_{0}}^{t_{0}+T} g(\xi, u, d) d t\right\}
$$

\section{Planning procedure}

A production planning algorithm has been developed, which consists of two parts: a supervisor and an operator. A Genetic Algorithm (GA) works as supervisor of the problem, by searching the DOFs that correspond to the maximum value of an objective function (fitness). The operator works within the supervisor, providing the operating parameters of the plant necessary for evaluating the fitness value. After having fixed DOFs, the operator performs its calculations and then the value of the fitness is evaluated.

The planning algorithm utilizes integers to account for the non-availability of a group and for the switching capability to put on-line or off-line a plant, relating to the convenience. Integers can be equal to 1 or 0 and the system of indexes is composed by:

- desired operating status index $d s_{j}$ of the $j$-th plant

- $\quad$ availability status index $\boldsymbol{a s}_{j}$ of the $j$-th plant

- actual operating status index $\boldsymbol{o s}_{j}$ of the $j$-th plant

Desired operating status indexes $\boldsymbol{d} \boldsymbol{s}$ establish what kind of operating status is desired for each plant of the pool at each time instant. Indexes os represent the actual statuses of plants, according to availabilities expressed by $\boldsymbol{a s}$ indexes.
Tab. 1 - Index relations

\begin{tabular}{|c|c|c|}
\hline $\begin{array}{c}\boldsymbol{d} \boldsymbol{s} \\
\text { Desired operating status }\end{array}$ & $\begin{array}{c}\boldsymbol{a s} \\
\text { Availability } \\
\text { status }\end{array}$ & $\begin{array}{c}\text { os } \\
\text { Actual operating status }\end{array}$ \\
\hline 0 & 0 & 0 \\
\hline 0 & 1 & 0 \\
\hline 1 & 0 & 0 \\
\hline 1 & 1 & 1 \\
\hline
\end{tabular}

The value 1 is associated to the on-line status, the value 0 is associated to the off-line status. Similarly, regarding to availability statuses, the value 1 is associated to the availability of the plant and the value 0 is associated to the unavailability of the plant. Tab. 1 shows how actual operating status indexes are evaluated starting by desired operating statuses and availabilities. The corresponding vectors are defined on the basis of the above indexes. Desired operating status indexes form the vector $\boldsymbol{D S}$ of the desired operating statuses. Availability status indexes form the vector $\boldsymbol{A S}$ of the availability statuses. Actual operating status indexes form the vector $\boldsymbol{O S}$ of the actual operating statuses.

The vectors introduced above are handled within the planning procedure by the supervisor routine, based on GA techniques. Fig. 3 explains how information involved in a planning problem is elaborated by the planning procedure. A starting random population of individuals is initialised. Each individual is a string of integer numbers, representing tentative values for operating statuses and allocated loads. The first half of the string contains operating status indexes, thus appears as a binary sequence of $0 \mathrm{~s}$ and $1 \mathrm{~s}$. The second half of the string is composed by allocated loads, reported as percentages of the nominal load. Values below the technical minimum operating limit are not allowed.

The dimension of the string depends on the number $J$ of plants considered for planning and the number $K$ of time intervals in which planning period is divided. Thus, the number of elements or genes that composes a string or individual is given by the following relation:

$$
D I M=2 \cdot J \cdot K
$$

Of course, the higher the dimension is, the longer the computation time. As approximate rule, the CPU occupancy for each generation is nearly proportional to the number of plants and time intervals.

Each string or individual forms the genotype, which represents a tentative solution or a potential planning. The phenotype has the same structure of genotype, but it represents an actual planning solution, compatible with availabilities of plant resources. The comparison between desired operating statuses and availability statuses is a part of the decoding process of genotype to phenotype.

As example, it could be considered the simple case of a pool composed by two power plants and a planning period divided into two time intervals only. In such a case, according to Eq. (8) strings or individuals are eight elements long and a string of the initial population would appear as follows:

\begin{tabular}{|l|l|l|l|l|l|l|l|}
0 & 1 & 1 & 1 & 80 & 76 & 70 & 95 \\
genotype
\end{tabular}

The above string represents a general genotype combination. As example, let's suppose that the first and third plants are unavailable, because of maintenance operations previously started. In addition, suppose that the second plant has passed nominal life for GT hot-section components, but it is still available (marked with red

\begin{tabular}{|c|c|c|c|c|c|c|c|c|}
\hline 0 & 1 & 1 & 1 & 80 & 76 & 70 & 95 & genotype \\
\hline$\nabla$ & $\nabla$ & 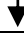 & 1 & & & & & \multirow{3}{*}{ availabilities } \\
\hline 0 & 1 & 0 & 1 & & & & & \\
\hline$\frac{V}{0}$ & $\nabla$ & $\nabla$ & $\nabla$ & & & & & \\
\hline 0 & 1 & 0 & 1 & 0 & 76 & 0 & 95 & phenotype \\
\hline
\end{tabular}
colour). According to index relations reported by Tab. 1, the phenotype will be evaluated as follows: 


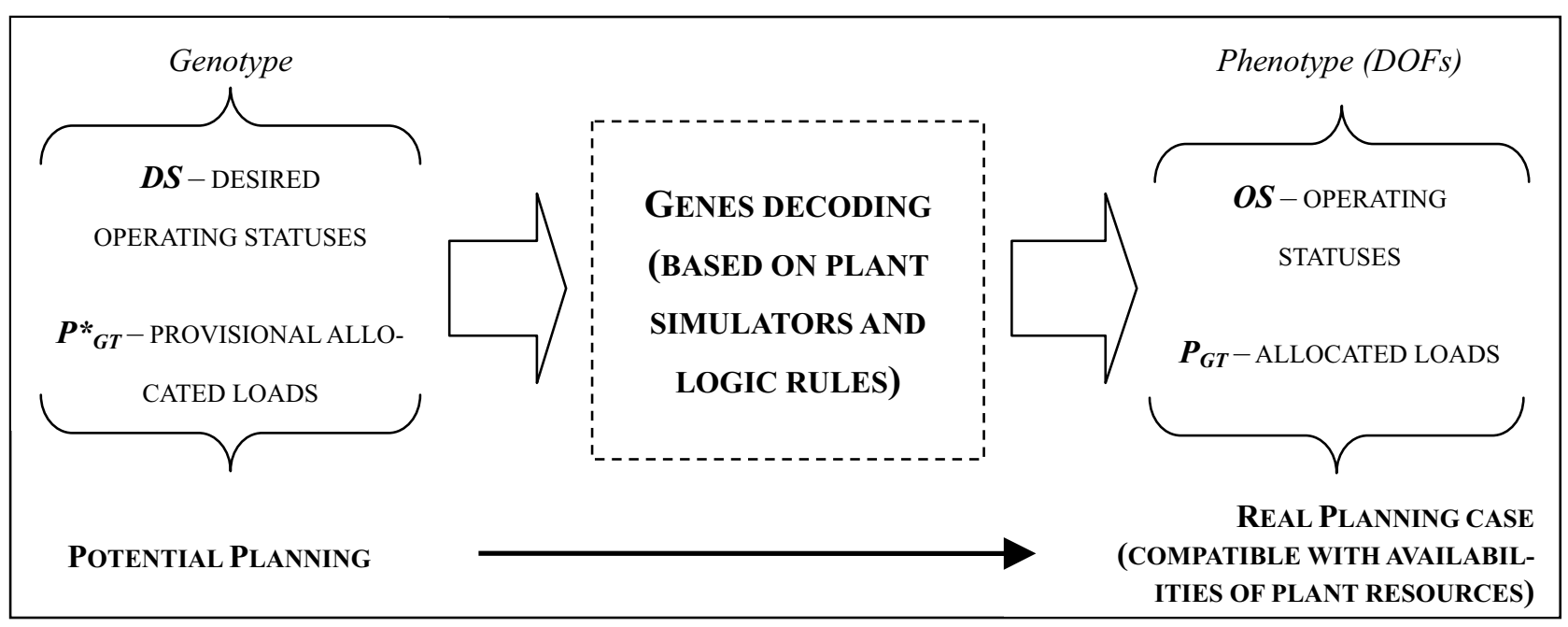

Fig. 3 - Integration of plant simulators within the planning algorithm based on Genetic Algorithms

Unavailable plants are put off-line. The fourth plant can be put on-line, because of no availability limitations. The second plant is kept on-line, but economic penalties are added to production costs to account for the risk of operating components over their nominal life. Allocated loads are adjusted according to values of actual operating statuses reported in the first half of the string.

Individual solutions are selected through a fitness-based process and solutions that better satisfy the fitness-function criteria are more likely to be selected. In order to evaluate the fitness function, genotype solutions (provisional) need to be converted to phenotype solutions, which take plant availabilities into account.

Since the solution of the problem is achieved by defining a discrete time-domain and writing Eq. (7) in finite terms, the fitness function is defined as follows:

$$
\text { Fitness }=\sum_{k=1}^{k_{\text {MAX }}}\left[R\left(t_{k}\right)-C\left(t_{k}\right)\right]
$$

$R\left(t_{k}\right)$ being the income during the time-interval $\Delta t_{k}$ :

$$
R\left(t_{k}\right)=p_{E L}\left(t_{k}\right) \cdot\left(\sum_{j=1}^{j_{M A X}} P_{G T, j}\left(t_{k}\right)+P_{S T, j}\left(t_{k}\right)\right) \cdot \Delta t_{k}
$$

$C\left(t_{k}\right)$ being the production cost during the time-interval $\Delta t_{k}$ :

$$
C\left(t_{k}\right)=\left(\sum_{j=1}^{j_{M A X}} C_{j}\left(t_{k}\right)\right) \cdot \Delta t_{k}
$$

and $p_{E L}$ being the sale price for power produced, $P_{G T, j}$ the power produced by the GT of the j-th plant, $P_{S T, j}$ the power produced by the steam turbine of the $j$-th plant and $C_{j}$ being the overall production costs. $\Delta t_{k}$ is the duration of k-th time interval $t_{k}$. Quantities needed for income or production costs evaluation, such as total net power output (comprehensive of steam turbine output level) or fuel consumption flow rate, are calculated by means of plant simulators during the decoding phase of genotype to phenotype.

Eq. (9) is the overall fitness function, which is extended to $k_{M A X}$ time intervals and to $j_{M A X}$ plants. The fitness function is constrained by the instantaneous behaviour of plant groups and by availabilities of each plant of the pool. As proposed by Cerri et al. (2000), such constraints can be given by plant simulators, which take actual statuses of components into account.

\section{RESULTS AND DISCUSSION}

The planning case presented focuses on the capability of managing postponement and anticipation of maintenance intervention to increase production profitability. The effects on production management of applying different damage-assessment techniques have been also investigated in a previous study and reported by Cerri and Gazzino (2006).

\section{Production planning case}

The planning case studied is defined as follows.

Boundary conditions are:

- Atmospheric conditions $\left(1^{\text {st }}, 2^{\text {nd }}, 3^{\text {rd }}, 5^{\text {th }}\right.$ plants $)$ $\mathrm{T}_{\mathrm{AMB}}=10^{\circ} \mathrm{C}, \mathrm{P}_{\mathrm{AMB}}=1013$ mbar, r.u. $60 \%$

- Atmospheric conditions ( $4^{\text {th }}$ plant): $\mathrm{T}_{\mathrm{AMB}}=15^{\circ} \mathrm{C}$, $\mathrm{P}_{\mathrm{AMB}}=1013$ mbar, r.u. $60 \%$

- Fuel price: $0.2 € / \mathrm{Nm} 3$

Initial HGPPs statuses are:

- $\quad 1$ st plant: $75 \%$ of equivalent life already used

- $\quad$ 2nd plant: $92 \%$ of equivalent life already used

- $\quad 3$ rd plant: New \& Clean condition $(0 \%)$

- $\quad$ 4th plant: New \& Clean condition $(0 \%)$

- $\quad$ 5th plant: New \& Clean condition $(0 \%)$

Power sale price distribution is reported in Fig. 9.

\section{Case solution}

Planning problem solution is reported in Fig. 4, Fig. 5, Fig. 6, Fig. 7 and Fig. 8. Each figure refers to a plant of the pool, starting from Fig. 4 associated to the first plant.

The power sale price distribution and the initial statuses of first and second plants have been properly chosen so as to highlight the postponement or anticipation capability of the planning algorithm. The power sale price increases its value before the middle of the planning period and then decreases rapidly at a value lower than the initial one. As a result, if maintenance is needed, it could be preferred to schedule the intervention when the power sale price has the lowest value of the period $T$, so as to keep the plant running when the price of electricity is high (i.e. between $0.2 \cdot T$ and $0.6 \cdot T$ ).

When a plant is put off-line a reduced income is suffered because of missing production. Thus, the number of maintenance interventions can actually affect final profit. Providing that service agreements are respected, a maintenance policy that includes postponement of maintenance interventions becomes profitable if costs connected with the risk of operating components over their equivalent life are lower than downtime costs on intervals that have power better priced. Similarly, the anticipation of maintenance intervention becomes profitable if costs connected with prematurely replacing HGPPs are recovered by the subsequent production availability. As example, such an occurrence can happen when an increase in power sale price is foreseen.

According to above, the planning algorithm:

1. Reduces loads (with respect to the instantaneous optimum) so as to reduce the damage rate and preserve hot components of the first plant.

2. Postpones maintenance intervention of the first plant.

3. The maintenance intervention on the first plant is scheduled when the power sale price is the lowest. 


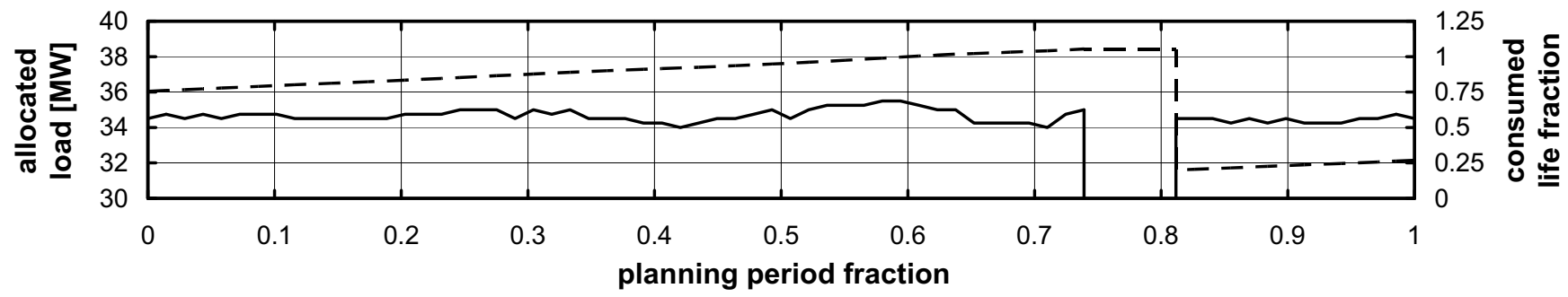

Fig. 4 - Allocated load on GT and consumed life fraction of HGPPs with reference to the first plant of the pool.

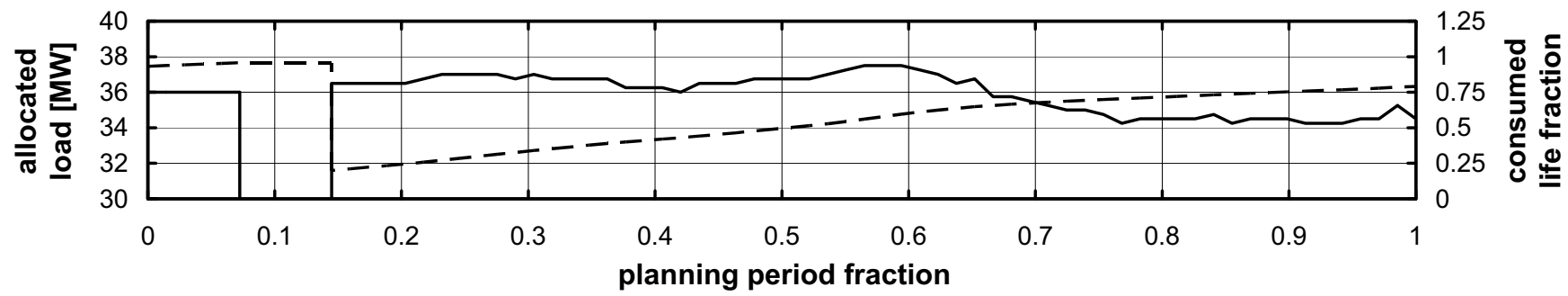

Fig. 5 - Allocated load on GT and consumed life fraction of HGPPs with reference to the second plant of the pool.

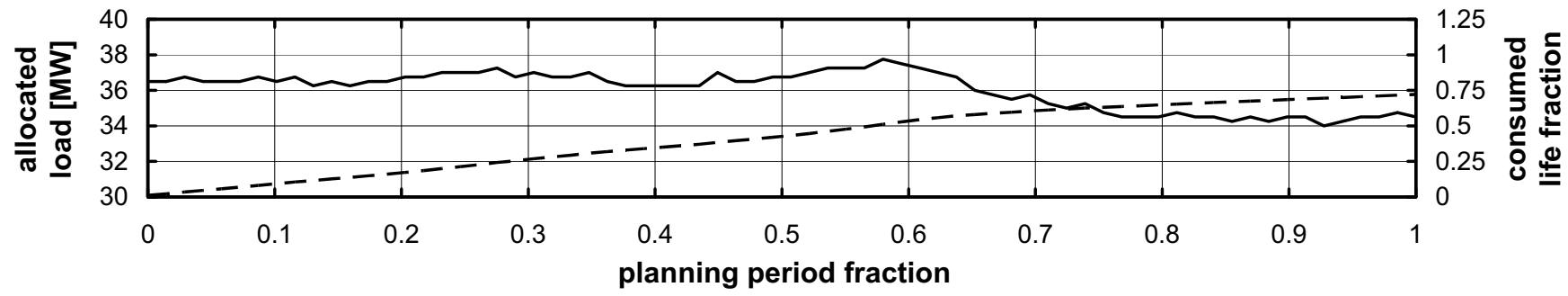

Fig. 6 - Allocated load on GT and consumed life fraction of HGPPs with reference to the third plant of the pool.

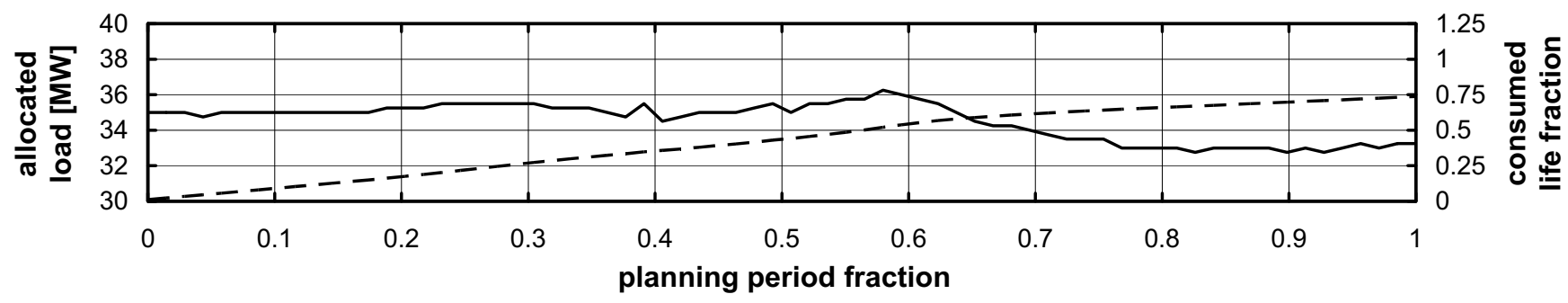

Fig. 7 - Allocated load on GT and consumed life fraction of HGPPs with reference to the fourth plant of the pool.

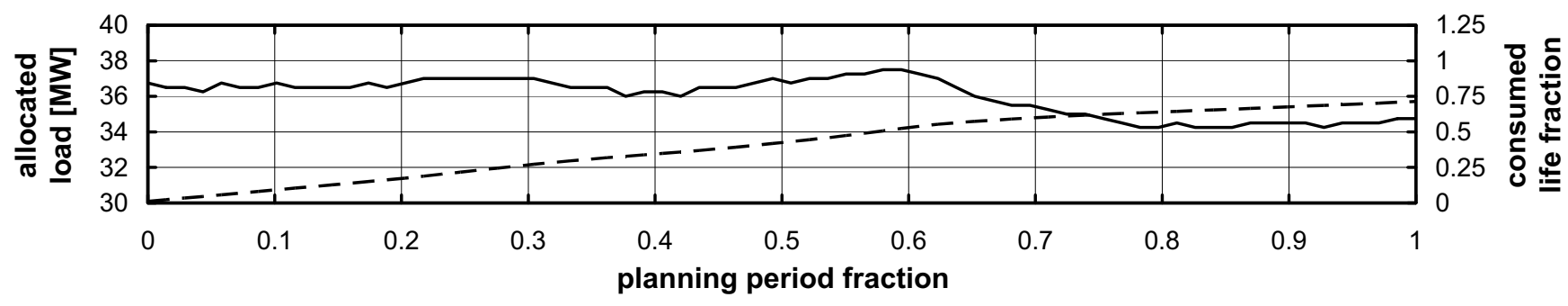

Fig. 8 - Allocated load on GT and consumed life fraction of HGPPs with reference to the fifth plant of the pool.

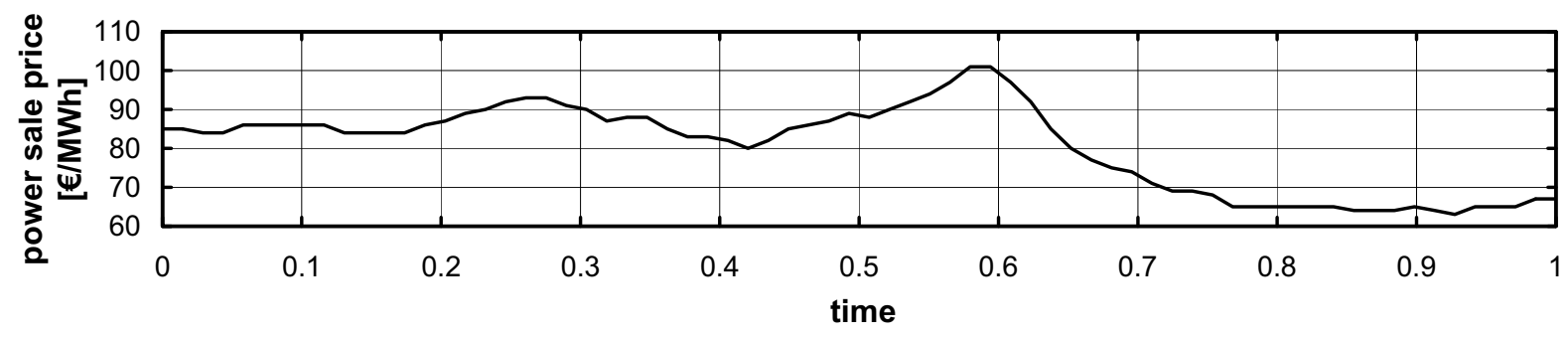

Fig. 9 - Sale price for power distribution. 


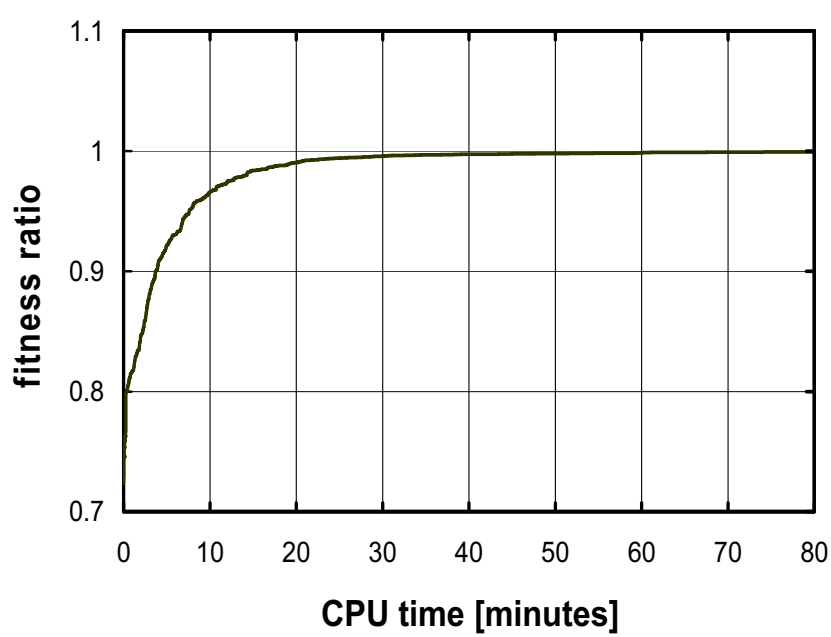

Fig. 10 - Convergence curve

4. The maintenance intervention on the second plant is anticipated at the $96 \%$ of the nominal equivalent life. This happened because initial status of hot components is too close to the nominal limit to allow postponement of maintenance. Costs connected with the prematurely replacing are recovered by the subsequent production availability over the interval with the highest power sale price.

5. The fourth plant shows loads allocated lower than the others because of the higher ambient temperature, which causes (at the same load) a corresponding higher deterioration cost of HGPPs.

In order to evaluate the time required to achieve the solution, the convergence curve has been drawn, as reported in Fig. 10. The $y$ axes reports the ratio between the fitness value at a generic calculation instant and the final value. Such a ratio has been named fitness ratio and it becomes equal to 1 when the solution is achieved.

The simulation and planning procedures have been run on an Intel Pentium IV based personal computer, with $2 \mathrm{GHz}$ of clock frequency, $1 \mathrm{MB}$ of resident cache and $1 \mathrm{~GB}$ of ram installed. Windows XP has been adopted as Operating System. All the routines have been written in Fortran 90 and compiled by a commercial developer kit, working at 16-32 bit.

As can be seen in figure, after thirty minutes from the start time of the planning routine, the fitness function has reached the $99,6 \%$ of its final value. The convergence is very rapid and ten minutes after having started the planning routine, the fitness function reached a value equal to $96 \%$.

\section{REFERENCES}

[1] BeÈr J.M., (2006), High Efficiency Electric Power Generation: the Environmental Role, Journal: Progress in Energy and Combustion Science, Elsevier, October, 2006, pp. 107-133

[2] BeaChKofSKi B.K., GrandHI R.V., (2004), Probabilistic System Reliability for a Turbine Engine Airfoil, ASME paper GT2004-53381, June 14-17, 2004, Vienna, Austria

[3] Benvenuti E., (2003), Innovative Gas Turbine Performance Diagnostics and Hot Parts Life Assessment Techniques, Proceedings of the $30^{\mathrm{TH}}$ turbo machinery symposium, 2003

[4] Cerri G., (1996), A Simultaneous Solution Method Based on a Modular Approach for Power Plant Analyses and Optimized Designs and Operations, ASME paper 96-GT-302, International Gas Turbine and Aeroengine Congress and Exhibition, Birmingham (UK), 10-13 June, 1996

\section{CONCLUSIONS}

The liberalization of electricity market has remarked the importance of optimizing power production, in order to perform an improved maintenance scheduling that takes market scenarios, as well as actual status and performance of plant components into account. In addition, the increasing concern for $\mathrm{CO}_{2}$ emissions pushes for improvements in power plant efficiencies, together with a more effective exploitation of energy and production resources. Such a policy becomes even more significant for resource-poor or energy-importer nations.

A key element to achieve the goals introduced above is the development of damage assessment and life prediction technologies. This paper has pointed out that damage models could be extremely useful as life assessment tools for the production planning and maintenance scheduling of a pool of GT based power plants. In addition, it has also shown that, as the turbine hot section is exposed to extreme operating conditions, the corresponding damage to the hot components plays a key role in maintenance management, cost optimization and production planning.

An economic benefit connected with the higher accuracy in estimating maintenance intervals may arise from the application of reliable damage models to plan power production. Such models make it possible to predict when hot-section life ends. Such knowledge is essential to evaluate the possibility of planning maintenance when sale price for power and the corresponding income are lower. In this manner, production resources can be better exploited and downtime costs minimized.

The application of alternative models for assessing life consumption of components allows providing with the following improvements:

1. Increased applicability to unit power outputs and ambient conditions different from baseline reference

2. Increased capability of being comprehensive of all operating conditions

The application of such models in conjunction with machine simulators provides with a tool that relates damage directly to the GT operating conditions, such as power level, ambient conditions, steam/water injection, air quality and so on. The manager of a pool of plants has a basis upon which to evaluate the consequences of different choices in production management. He can maximize profit, if it is a main concern, or he can tune power output according to a life consumption level, by considering, on the basis of creep model assessment, the effect of saving the life of components. In this manner, the economic impact of extended-life operations can be easily evaluated. Similarly, the over-damage costs connected with peaking operations can be assessed.

\section{ACKNOWLEDGEMENTS}

Thanks are due to Prof. Lorenzo Battisti for his useful advice on methodologies adopted for creep modelling. Support from MIUR is also acknowledged.

[5] Cerri G., Borghetti S., Salvini C., (2006), Models for Simulation and Diagnosis of Energy Plant Components, ASME Paper PWR2006-88146, ASME Power, Atlanta, Georgia, USA, 2-4 May, 2006

[6] Cerri G., Borghetti S., Salvini C., (2005-a), Inverse Methodologies for Actual Status Recognition of Gas Turbine Components, ASME Paper PWR2005-50033, ASME Power, Chicago, Illinois, USA, 5-7 April, 2005

[7] Cerri G., Borghetti S., Salvini C., (2005-b), Neural Management for Heat and Power Cogeneration Plants, The 9th International Conference on Engineering Applications of Neural Networks EANN2005, Lille, France, 24-26 August 2005 
[8] Cerri G., Evans R., Frangopoulos C.A., Parrella M., Pitt R.U., Psychogios J., Salvador S., Sepielli M., SEYEDAN B., (2000), Optimum Management System with Environmental Monitoring, ASME paper 2000-GT-310, ASME Turbo Expo 2000, Monaco, D, May 8-11, 2000

[9] Silva P., Campanari S., MaCChi E., (2003), Optimization of Operating Conditions and Compressor Cleaning Time Intervals of Combined Cycles in a Liberalized Market, Proceedings of ASME Turbo Expo, June 16-19, Atlanta, Georgia, USA

[10] Cunha F.J., DAhmer M.T., Chyu M.K., (2005), Thermal-Mechanical Life Prediction System for Anisotropic Turbine Components, ASME paper GT2005-68107, June 6-9, 2005, Reno-Tahoe, Nevada

[11] Cerri G., Gazzino M., Borghetti S., (2006), Hot Section Life Assessment by a Creep Model to Plan Gas Turbine Based Power Plant Electricity Production, The Future of Gas Turbine Technology, $3^{\text {rd }}$ International Conference, 11-12 October 2006, Brussels, Belgium

[12] Gazzino M., (2005), Influenza del Creep dei Componenti ad Alta Temperatura sulla Pianificazione della Produzione Elettrica di un Parco di Centrali Equipaggiate con Turbina a Gas, Master Degree Thesis, Roma Tre University, 2005

[13] HeE N.B., IDRIS Z., (2000), Managing the Risk of Extended EOH of ABB Gas Turbines at Connaught Bridge Power Station, Connaught Bridge Power Station, Maintenance \& Engineering Unit, Klang, Selangor, Malaysia 2000

[14] Hoeft R., Janawitz J., KeCK R., (2003), Heavy-Duty Gas Turbine Operative and Maintenance Considerations, GE Power Generation Report GER3620J, 2003

[15] LEE J.H., (1999), Total Operating Activities of Gas Turbine Components - Total Equivalent Operating Hours (TEOH), ASME 99-GT-244, June 7-10, 1999, Indianapolis, Indiana

[16] Liu Z., Mavris D.N., Volovoi V.V., (2001), Creep Life Prediction of a Gas Turbine Components under Varying Operating Conditions, Proceedings of 2001 ASME International Joint Power Generation Conference, June 4-7, 2001, New Orleans, Louisiana, USA

[17] Lowden P., Weber B., Grant R., Jin H., Pistor R., (2006),Prediction of Gas Turbine Blade Life: An Interdisciplinary Engineering Approach for Condition-Based Maintenance, ASME paper GT2006-90037, May 8-11, 2006, Barcelona, Spain
[18] Ross E.W., O'Hara K.S., (1992), Renè 142: A High Strength, Oxidation Resistant DS Turbine Airfoil Alloy, Proceedings of $7^{\mathrm{TH}}$ International Symposium on Superalloys in Champion, Pennsylvania, September 20-24, 1992, pp. 257-266

[19] Sampath S., SingH R., (2003), Managing Maintenance and Operational Availability of Gas Turbine Propelled Naval Ships Through Advanced Engine Fault Diagnostics, paper GT2003-38456, Proceedings of ASME TURBO EXPO 2003, June 16-19, 2003, Atlanta, Georgia, USA

[20] Wallace J.M., Mavris D.N., (2003), Creep Life Uncertainty Assessment of a Gas Turbine Airfoil, Proceedings of 5th AIAA Non-Deterministic Approaches Forum, Norfolk, VA, April 7-10, 2003

[21] On-Site Operation and Maintenance Manual for General Electric LM6000-PA Series Gas Turbines, GE company, GEK-98493, Vol. I

[22] Viswanathan R., Cheruvu N.S., Chan K.S., (2003), Coatings for Advanced Large Frame Combustion Turbines for Power Generation, Proceedings of ASME TURBO EXPO 2003, June 16-19, 2003, Atlanta, Georgia, USA

[23] Chan K.S., Cheruvu N.S., Leverant G.R., Viswanathan R., (2003), Life Prediction Strategies for Land-Based Gas Turbine Blades, Proceedings of the 2003 TMS Annual Meeting \& Exhibition, San Diego, California, March 2-6, 2003, pp. 191-209

[24] Wallace J.M., MaVris D.N., (2003), Creep Life Uncertainty Assessment of a Gas Turbine Airfoil, Proceedings of 5th AIAA Non-Deterministic Approaches Forum, Norfolk, VA, April 7-10, 2003

[25] LiU Z., Mavris D.N., Volovoi V.V., (2001), Creep Life Prediction of a Gas Turbine Components under Varying Operating Conditions, Proceedings of 2001 ASME International Joint Power Generation Conference, June 4-7, 2001, New Orleans, Louisiana, USA 